\title{
New Integrals Arising in the Samara-Valencia Heat Transfer Model in Grinding
}

\author{
J. L. González-Santander \\ Universidad Católica de Valencia "San Vicente Mártir", C/Guillem de Castro 94, 46001 Valencia, Spain \\ Correspondence should be addressed to J. L. González-Santander; martinez.gonzalez@ucv.es
}

Received 20 August 2017; Accepted 24 October 2017; Published 14 November 2017

Academic Editor: Ali R. Ashrafi

Copyright ( 2017 J. L. González-Santander. This is an open access article distributed under the Creative Commons Attribution License, which permits unrestricted use, distribution, and reproduction in any medium, provided the original work is properly cited.

The Samara-Valencia model for heat transfer in grinding has been recently used for calculating nontabulated integrals. Based on these results, new infinite integrals can be calculated, involving the Macdonald function and the modified Struve function.

\section{Introduction}

Usually, mathematical developments facilitate the computation of mathematical modeling expressions in many different fields. However, mathematical modeling also yields in many cases a good field to develop new mathematical identities and formulas. This is the case of the mathematical modeling of heat transfer in surface grinding. This machining process consists in material removal from a workpiece by an abrasive wheel that rotates at high speed over its surface [1]. Classically, Jaeger's model $[2,3]$ is used for the calculation of the temperature field in dry grinding. DesRuisseaux's model [4] extends Jaeger's model to include the effect of surface cooling (wet grinding). More recently, the SamaraValencia model [5] has been proposed. In this model, the twodimensional convective heat equation is considered. Also, the heat flux profile entering the workpiece and the action of the coolant are considered in the boundary condition. In [5], this boundary-value problem is transformed into an integral equation that is useful for the numerical evaluation of the heat transfer in intermittent wet grinding [6]. However, in the case of dry grinding, this integral equation can be reduced to a two-dimensional integral $\left(T^{(0)}\right.$ theorem $)$ [7].

New mathematical identities have been proved in this framework. For instance, comparing Jaeger's model with Samara-Valencia model, a new Dirac delta representation [8],

$$
\lim _{u \rightarrow 0} \frac{|u|}{\pi} \frac{K_{1}\left(\sqrt{x^{2}+u^{2}}\right)}{\sqrt{x^{2}+u^{2}}}=\delta(x),
$$

and two new nontabulated integrals [9] have been obtained. Also, by using $T^{(0)}$ theorem, the following integrals have been calculated [10]:

$$
\begin{aligned}
& \int_{0}^{\infty} \cosh (\alpha \xi) K_{0}\left(\beta \sqrt{\xi^{2}+y^{2}}\right) d \xi \\
& =\frac{\pi \exp \left(-|y| \sqrt{\beta^{2}-\alpha^{2}}\right)}{2 \sqrt{\beta^{2}-\alpha^{2}}}, \quad \beta>|\alpha|, \quad y \in \mathbb{R}, \\
& K_{0}\left(\sqrt{x^{2}+y^{2}}\right) \\
& \quad=\frac{|y|}{\pi} \int_{-\infty}^{\infty} K_{0}(|\xi-x|) \frac{K_{1}\left(\sqrt{\xi^{2}+y^{2}}\right)}{\sqrt{\xi^{2}+y^{2}}} d \xi, \\
& \int_{-\infty}^{\infty} K_{0}(|\xi|) K_{0}(|x-\xi|) d \xi=\frac{\pi^{2}}{2} e^{-|x|}, \quad x \in \mathbb{R} .
\end{aligned}
$$

It is worth noting that (3) has been calculated also in [9] by using a complex integration contour. Recently, in [11], the following generalization of (4) has been calculated as a finite sum of terms containing beta and hypergeometric functions, 
by using the convolution theorem of the Fourier transform. This generalization reads as follows:

$$
\begin{aligned}
& \int_{-\infty}^{\infty}\left|t^{\prime}\right|^{\alpha+2 n} K_{\alpha}\left(a\left|t^{\prime}\right|\right)\left|t-t^{\prime}\right|^{\beta+2 m} K_{\beta}\left(a\left|t-t^{\prime}\right|\right) d t^{\prime} \\
& =\frac{\pi}{a} \frac{(2 n) !(2 m) !}{(2 a)^{\mu}} \Gamma(2 \alpha+2 n+1) \Gamma(2 \beta+2 m+1) \\
& \times \sum_{k=0}^{n+m}(-4)^{k} c_{k}(n, m, \alpha, \beta) \times\left\{\frac{1}{2}\right. \\
& B\left(k+\frac{1}{2}, \mu-k+\frac{1}{2}\right) \\
& \cdot{ }_{1} F_{2}\left(\begin{array}{c|c}
k+\frac{1}{2} & \frac{a^{2} t^{2}}{4} \\
\frac{1}{2}, k-\mu+\frac{1}{2} &
\end{array}\right)+(-1)^{k+1} \\
& \cdot|a t|^{2(\mu-k)+1} \sin \pi(\alpha+\beta) \Gamma(2(k-\mu)-1) \\
& \left.\times{ }_{1} F_{2}\left(\begin{array}{c|c}
\mu+1 \\
\mu-k+1, \mu-k+\frac{3}{2} & \frac{a^{2} t^{2}}{4}
\end{array}\right)\right\} \text {, } \\
& \operatorname{Re} \alpha>-\frac{1}{2}-n, \operatorname{Re} \beta>-\frac{1}{2}-m, a>0, t \in \mathbb{R},
\end{aligned}
$$

where the following coefficients are defined as

$$
c_{k}(n, m, \alpha, \beta)=\sum_{l=\max (k-m, 0)}^{\min (n, k)} b_{l}(n, \alpha) b_{k-l}(m, \beta),
$$

with

$$
b_{l}(n, \alpha)=\frac{1}{(n-l) !(2 l) ! \Gamma(n+\alpha-l+1)} .
$$

The scope of this paper is just to calculate more integrals based on results (2)-(4), which do not seem to be reported in the most common tables of integrals [12-14].

This paper is organized as follows. Section 2 is devoted to the calculation of the new integrals. It is divided into three subsections, each one of them containing one new result. Section 3 collects the conclusions, highlighting the main results obtained in the body of the paper.

\section{The Integrals}

\subsection{First Result}

Theorem 1. The following integral holds true:

$$
\begin{aligned}
& \int_{-\infty}^{\infty} e^{ \pm \alpha \xi} \frac{K_{1}\left(\beta \sqrt{\xi^{2}+y^{2}}\right)}{\sqrt{\xi^{2}+y^{2}}} d \xi \\
& =2 \int_{0}^{\infty} \cosh (\alpha \xi) \frac{K_{1}\left(\beta \sqrt{\xi^{2}+y^{2}}\right)}{\sqrt{\xi^{2}+y^{2}}} d \xi \\
& =\frac{\pi \exp \left(-|y| \sqrt{\beta^{2}-\alpha^{2}}\right)}{\beta|y|}, \quad \beta>|\alpha|, y \in \mathbb{R} \backslash\{0\} .
\end{aligned}
$$

Proof. Expanding the hyperbolic cosine as $\cosh x=\left(e^{x}+\right.$ $\left.e^{-x}\right) / 2[15$, Eqn. 8.2] in (2), we have

$$
\begin{gathered}
\frac{\pi \exp \left(-|y| \sqrt{\beta^{2}-\alpha^{2}}\right)}{\sqrt{\beta^{2}-\alpha^{2}}} \\
=\int_{0}^{\infty} e^{\alpha \xi} K_{0}\left(\beta \sqrt{\xi^{2}+y^{2}}\right) d \xi \\
\quad+\int_{0}^{\infty} e^{-\alpha \xi} K_{0}\left(\beta \sqrt{\xi^{2}+y^{2}}\right) d \xi .
\end{gathered}
$$

Now, perform the change of variables $\xi \rightarrow-\xi$, and rewrite the first or the second integral given in (9) as

$$
\begin{aligned}
& \int_{0}^{\infty} e^{ \pm \alpha \xi} K_{0}\left(\beta \sqrt{\xi^{2}+y^{2}}\right) d \xi \\
& \quad=\int_{-\infty}^{0} e^{\mp \alpha \xi} K_{0}\left(\beta \sqrt{\xi^{2}+y^{2}}\right) d \xi .
\end{aligned}
$$

Therefore, inserting (10) in (9), we can define the following function:

$$
\begin{aligned}
F(y) & =\int_{-\infty}^{\infty} e^{ \pm \alpha \xi} K_{0}\left(\beta \sqrt{\xi^{2}+y^{2}}\right) d \xi \\
& =\frac{\pi \exp \left(-|y| \sqrt{\beta^{2}-\alpha^{2}}\right)}{\sqrt{\beta^{2}-\alpha^{2}}}, \quad \beta>|\alpha|, \quad y \in \mathbb{R} .
\end{aligned}
$$

Let us take temporarily $y>0$, so that we can drop the absolute value in (11). Then, knowing that $K_{0}^{\prime}(x)=-K_{1}(x)[16$, Eqn. 51:10:2], we have

$$
\begin{aligned}
F^{\prime}(y) & =\beta y \int_{-\infty}^{\infty} e^{ \pm \alpha \xi} \frac{K_{1}\left(\beta \sqrt{\xi^{2}+y^{2}}\right)}{\sqrt{\xi^{2}+y^{2}}} d \xi \\
& =\pi \exp \left(-y \sqrt{\beta^{2}-\alpha^{2}}\right) .
\end{aligned}
$$

Recover now the absolute value and take into account again (9)-(10) to obtain (8).

Remark 2. It is worth noting that when $y=0$, (12) seems to fail. Nonetheless, this is apparent. In order to see it, rewrite the RHS of (12) as follows, knowing that $\beta>0$ and performing the changes of variables $x=\beta x$ and $u=\beta y$ :

$$
F^{\prime}(u)=u \int_{-\infty}^{\infty} \exp \left( \pm \frac{\alpha}{\beta} x\right) \frac{K_{1}\left(\sqrt{x^{2}+u^{2}}\right)}{\sqrt{x^{2}+u^{2}}} d x .
$$

Therefore, taking the limit $u \rightarrow 0$ and applying the Dirac delta representation given in (1), we have

$$
\begin{aligned}
& \lim _{u \rightarrow 0} F^{\prime}(u) \\
& =\lim _{u \rightarrow 0} u \int_{-\infty}^{\infty} \exp \left( \pm \frac{\alpha}{\beta} x\right) \frac{K_{1}\left(\sqrt{x^{2}+u^{2}}\right)}{\sqrt{x^{2}+u^{2}}} d x \\
& \quad=\pi \int_{-\infty}^{\infty} \exp \left( \pm \frac{\alpha}{\beta} x\right) \delta(x) d x=\pi,
\end{aligned}
$$


which agrees with the LHS of (12), performing the limit $y \rightarrow$ 0 .

We can continue calculating derivatives with respect to $y$ in (12) in order to get new integrals, but the integrands we get are increasingly complex and we omit these results here.

\subsection{Second Result}

Theorem 3. The following integral holds true:

$$
\begin{aligned}
& \int_{0}^{\infty} K_{0}(\xi) K_{0}\left(\sqrt{\xi^{2}+y^{2}}\right) d \xi=\frac{\pi^{2}}{4}\{1 \\
& \left.-|y|\left[K_{0}(|y|) \mathbf{L}_{-1}(|y|)+K_{1}(|y|) \mathbf{L}_{0}(|y|)\right]\right\},
\end{aligned}
$$

$$
y \in \mathbb{R} .
$$

Proof. Let us define

$$
\begin{aligned}
G(y) & =\int_{0}^{\infty} K_{0}(\xi) K_{0}\left(\sqrt{\xi^{2}+y^{2}}\right) d \xi \\
& =\frac{1}{2} \int_{-\infty}^{\infty} K_{0}(|\xi|) K_{0}\left(\sqrt{\xi^{2}+y^{2}}\right) d \xi
\end{aligned}
$$

Notice that

$$
G(y)=G(|y|)
$$

thereby, hereafter, we will assume that $y>0$, dropping the absolute value. Performing in (17) the derivative with respect to $y$, we have

$$
G^{\prime}(y)=-\frac{y}{2} \int_{-\infty}^{\infty} K_{0}(|\xi|) \frac{K_{1}\left(\sqrt{\xi^{2}+y^{2}}\right)}{\sqrt{\xi^{2}+y^{2}}} d \xi .
$$

The above integral can be calculated taking $x=0$ in (3); thus,

$$
G^{\prime}(y)=-\frac{\pi}{2} K_{0}(y)
$$

Now, let us apply the following integral [12, Eqn. 1.12.1(3)]:

$$
\begin{aligned}
& \int_{0}^{z} x^{\nu} K_{v}(x) d x=2^{\nu-1} \sqrt{\pi} \Gamma\left(v+\frac{1}{2}\right) \\
& \cdot z\left[K_{v}(z) \mathbf{L}_{v-1}(z)+K_{\nu-1}(z) \mathbf{L}_{v}(z)\right],
\end{aligned}
$$

where $\mathbf{L}_{\nu}(z)$ is the modified Struve function, defined as [17, Eqn. 11.2.2]

$$
\mathbf{L}_{\nu}(z)=\sum_{n=0}^{\infty} \frac{(z / 2)^{2 n+v+1}}{\Gamma(n+3 / 2) \Gamma(n+\nu+3 / 2)} .
$$

Therefore, taking in (21) $v=0$, knowing that $K_{-v}(z)=K_{v}(z)$ ([18], Eqn. 5.7.10) and also that [16, Eqn. 43:4:2]

$$
\Gamma\left(\frac{1}{2}\right)=\sqrt{\pi}
$$

we calculate $G(y)$ from (20) as

$$
G(y)=-\frac{\pi}{4} y\left[K_{0}(y) \mathbf{L}_{-1}(y)+K_{1}(y) \mathbf{L}_{v}(y)\right]+C
$$

where $C$ is an integration constant. In order to calculate this integration constant, notice, on the one hand, that, from (16), we have

$$
G(0)=\int_{0}^{\infty} K_{0}^{2}(\xi) d \xi
$$

The above integral can be calculated taking $x=0$ in (4):

$$
\int_{-\infty}^{\infty} K_{0}^{2}(|\xi|) d \xi=2 \int_{0}^{\infty} K_{0}^{2}(\xi) d \xi=\frac{\pi^{2}}{2} .
$$

Thus,

$$
G(0)=\frac{\pi^{2}}{4}
$$

On the other hand, taking limits in (24), we have

$$
\begin{aligned}
G(0)= & C \\
& -\frac{\pi}{4} \lim _{y \rightarrow 0} y\left[K_{0}(y) \mathbf{L}_{-1}(y)+K_{1}(y) \mathbf{L}_{0}(y)\right] .
\end{aligned}
$$

According to (22), consider the following asymptotic formula:

$$
\mathbf{L}_{-1}(y) \approx \frac{1}{\Gamma(3 / 2) \Gamma(1 / 2)}=\frac{2}{\pi}, \quad y \longrightarrow 0,
$$

where we have applied the property of the gamma function $\Gamma(z+1)=z \Gamma(z)$ ([18], Eqn. 1.2.1) and (23). Similarly, we have

$$
\mathbf{L}_{0}(y) \approx \frac{y / 2}{\Gamma(3 / 2) \Gamma(1 / 2)}=\frac{y}{\pi}, \quad y \rightarrow 0 .
$$

Also, the asymptotic behavior of the Macdonald function for $y \rightarrow 0$ is ([17], Eqn. 10.30.2-3)

$$
\begin{aligned}
& K_{0}(y) \approx-\log y, \\
& K_{\nu}(y) \approx \frac{1}{2} \Gamma(\nu)\left(\frac{y}{2}\right)^{-v}, \quad \operatorname{Re} v>0,
\end{aligned}
$$

and thus

$$
K_{1}(y) \approx \frac{1}{y}
$$

Therefore, taking into account (29)-(32), we calculate the limit given in (28) as

$$
\begin{gathered}
\lim _{y \rightarrow 0} y\left[K_{0}(y) \mathbf{L}_{-1}(y)+K_{1}(y) \mathbf{L}_{0}(y)\right] \\
=\frac{1}{\pi} \lim _{y \rightarrow 0} y[1-2 \log y]=0 .
\end{gathered}
$$

Thereby, inserting (33) in (28) and recalling (27), we calculate the integration constant as follows:

$$
G(0)=C=\frac{\pi^{2}}{4}
$$

Finally, substituting (34) into (24) and remembering the definition of $G(y)$, we arrive at (15), where we have considered (18). 


\subsection{Third Result}

Theorem 4. The next integral holds true:

$$
\int_{0}^{\infty}\left\{1-y\left[K_{0}(y) \mathbf{L}_{-1}(y)+K_{1}(y) \mathbf{L}_{0}(y)\right]\right\} d y=\frac{2}{\pi} .
$$

Proof. For the third result, integrate both sides of (16) as follows:

$$
\begin{aligned}
& \int_{0}^{\infty} G(y) d y \\
& \quad=\int_{0}^{\infty} K_{0}(\xi)\left\{\int_{0}^{\infty} K_{0}\left(\sqrt{\xi^{2}+y^{2}}\right) d y\right\} d \xi .
\end{aligned}
$$

In order to calculate the inner integral given in (36), consider $\alpha=0$ and $\beta=1$ in (2); hence,

$$
\int_{0}^{\infty} K_{0}\left(\sqrt{\xi^{2}+y^{2}}\right) d y=\frac{\pi}{2} e^{-|\xi|}
$$

Inserting (37) into (36), we can drop the absolute value in the exponential, since the variable of integration is positive (i.e., $\xi \in(0, \infty))$; thereby,

$$
\int_{0}^{\infty} G(y) d y=\frac{\pi}{2} \int_{0}^{\infty} K_{0}(\xi) e^{-\xi} d \xi .
$$

To calculate the above integral, in the literature, we find the following integral, termed King's integral [19, Eqn. 11.3.16]:

$$
\int_{0}^{x} e^{ \pm u} K_{0}(u) d u=x e^{ \pm x}\left[K_{0}(x) \pm K_{1}(x)\right] \mp 1
$$

Hence, applying to King's Integral and the asymptotic formula [18, Eqn. 5.16.5]

$$
K_{\nu}(x) \approx \sqrt{\frac{\pi}{2 x}} e^{-x}, \quad x \longrightarrow+\infty
$$

we obtain

$$
\begin{aligned}
\int_{0}^{\infty} K_{0}(\xi) e^{-\xi} d \xi & =\lim _{x \rightarrow \infty} x e^{-x}\left[K_{0}(x)-K_{1}(x)\right]+1 \\
& =1 .
\end{aligned}
$$

Recalling now the result given in (15) for $G(y)$ and taking into account (41), (38) finally reads as (35).

\section{Conclusions}

Based on integrals (2)-(4), calculated in the framework of the Samara-Valencia heat transfer model in surface grinding by using the $T^{(0)}$ theorem, new integrals have been derived. From (2), we have derived integrals (11) and (8). Also, applying (3) and (4), we have derived (15). Finally, integrating the result given in (15) and taking into account (2), the integral given in (35) has been obtained. It is worth noting that these results have been confirmed, evaluating numerically the corresponding integrals.

\section{Conflicts of Interest}

The author declares that there are no conflicts of interest regarding the publication of this paper.

\section{Acknowledgments}

The author wishes to acknowledge the financial support received from Universidad Católica de Valencia under Grants PRUCV/2015/612 and 2017-160-001.

\section{References}

[1] S. Malkin and C. Guo, Grinding Technology: Theory and Application of Machining with Abrasives, Industrial Press, New York, NY, USA, 2008.

[2] J. C. Jaeger, "Moving sources of heat and the temperature at sliding contacts," Journal and Proceedings of the Royal Society of New South Wales, vol. 76, pp. 204-224, 1942.

[3] H. S. Carslaw and J. C. Jaeger, Conduction of Heat in Solids, Oxford Science Publications, 1995.

[4] N. R. DesRuisseaux and R. D. Zerkle, "Temperature in semiInfinite and cylindrical bodies subjected to moving heat surfaces and surface Cooling," Journal of Heat Transfer, vol. 92, no. 3, pp. 456-464, 1970.

[5] D. L. Skuratov, Y. L. Ratis, I. A. Selezneva, J. Pérez, P. F. de Córdoba, and J. F. Urchueguía, "Mathematical modelling and analytical solution for workpiece temperature in grinding," Applied Mathematical Modelling, vol. 31, no. 6, pp. 1039-1047, 2007.

[6] J. Pérez, S. Hoyas, D. L. Skuratov et al., "Heat transfer analysis of intermittent grinding processes," International Journal of Heat and Mass Transfer, vol. 51, no. 15-16, pp. 4132-4138, 2008.

[7] J. L. González-Santander, J. M. Valdés Placeres, and J. M. Isidro, "Exact solution for the time-dependent temperature field in dry grinding: application to segmental wheels," Mathematical Problems in Engineering, vol. 2011, Article ID 927876, 28 pages, 2011.

[8] J. L. González-Santander, J. Pérez, P. Fernández de Córdoba, and J. M. Isidro, "An analysis of the temperature field of the workpiece in dry continuous grinding," Journal of Engineering Mathematics, vol. 67, no. 3, pp. 165-174, 2009.

[9] J. L. G. Santander, P. Castañeda Porras, Y. L. Ratis, J. M. Isidro, and P. Fernández De Córdoba, "Calculation of some integrals arising in heat transfer in grinding," Mathematical Problems in Engineering, vol. 2010, Article ID 535801, 14 pages, 2010.

[10] J. L. González-Santander, "Calculation of some integrals arising in the samara-valencia solution for dry flat grinding," Mathematical Problems in Engineering, vol. 2015, Article ID 428461, 7 pages, 2015.

[11] J. L. Santander, "Calculation of some integrals involving the Macdonald function by using Fourier transform," Journal of Mathematical Analysis and Applications, vol. 441, no. 1, pp. 349363, 2016.

[12] A. P. Prudnikov, Y. A. Brychkov, and O. I. Marichev, Integrals and Series, Vol. 2: Special Functions, Gordon and Breach Science Publishers, New York, NY, USA, 1986.

[13] A. P. Prudnikov, Y. A. Brychkov, and O. I. Marichev, Integrals and Series, Vol. 3: More Special Functions, Gordon and Breach Science Publishers, New York, NY, USA, 1986. 
[14] I. S. Gradshteyn and I. M. Ryzhik, Table of Integrals, Series and Products, Academic Press Inc., New York, NY, USA, 7th edition, 2007.

[15] M. R. Spiegel, Handbook of Mathematical Formulas, SchaumMcGraw Hill, New York, NY, USA, 1968.

[16] K. Oldham, J. Myland, and J. Spanier, An Atlas of Functions, Springer, New York, NY, USA, 2nd edition, 2008.

[17] F. W. J. Olver, D. W. Lozier, R. F. Boisvert, and C. W. Clark, NIST Handbook of Mathematical Functions, Cambridge University Press, New York, NY, USA, 2010.

[18] N. N. Lebedev, Special Functions and Their Applications, Dover Publications, New York, NY, USA, 1972.

[19] M. Abramowitz and I. A. Stegun, Handbook of Mathematical Functions, Dover Publications, Wahshington, DC, USA, 1972. 


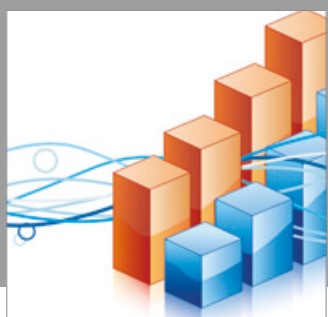

Advances in

Operations Research

vatersals

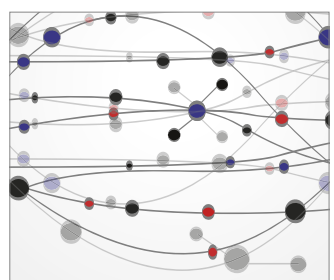

\section{The Scientific} World Journal
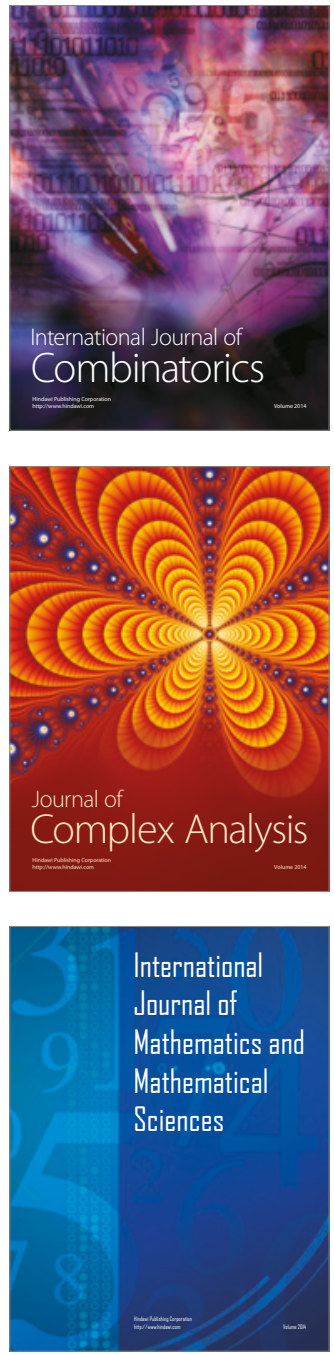
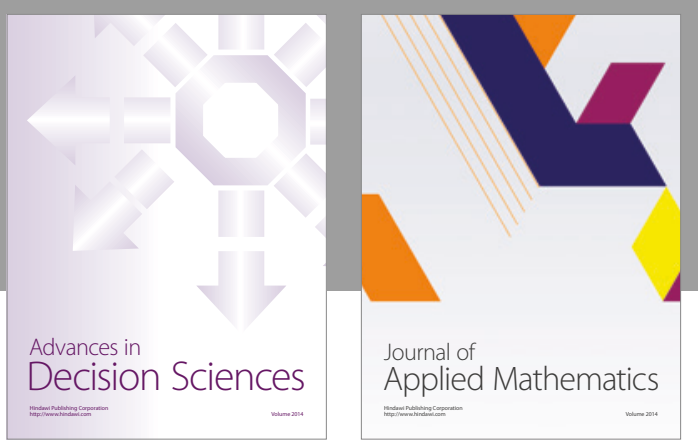

Algebra

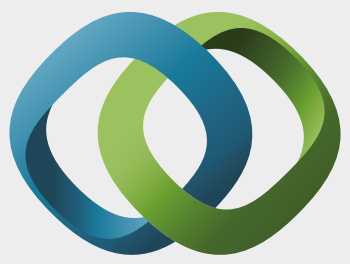

\section{Hindawi}

Submit your manuscripts at

https://www.hindawi.com
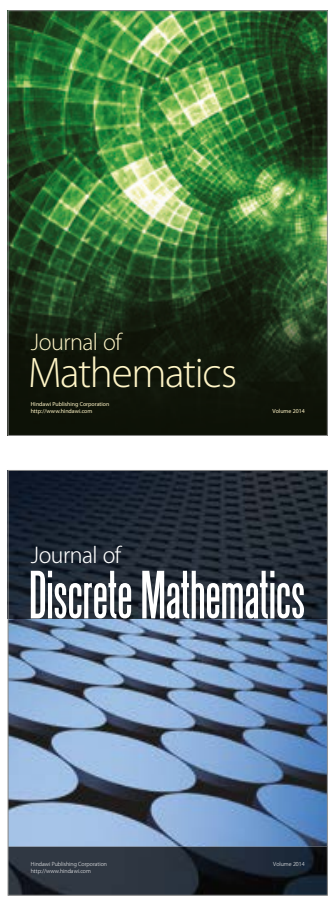

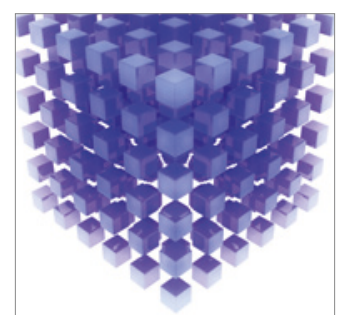

Mathematical Problems in Engineering
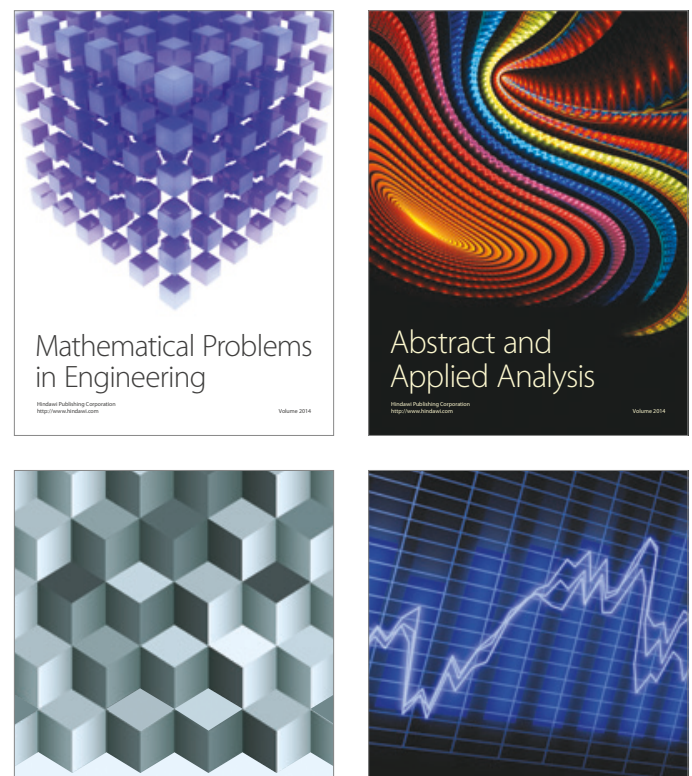

Journal of

Function Spaces

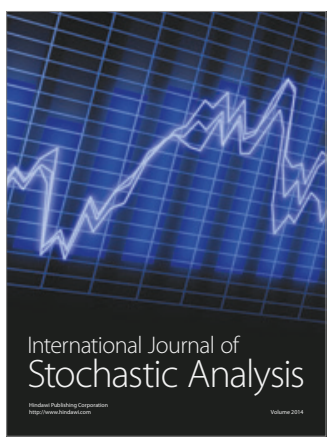

Probability and Statistics
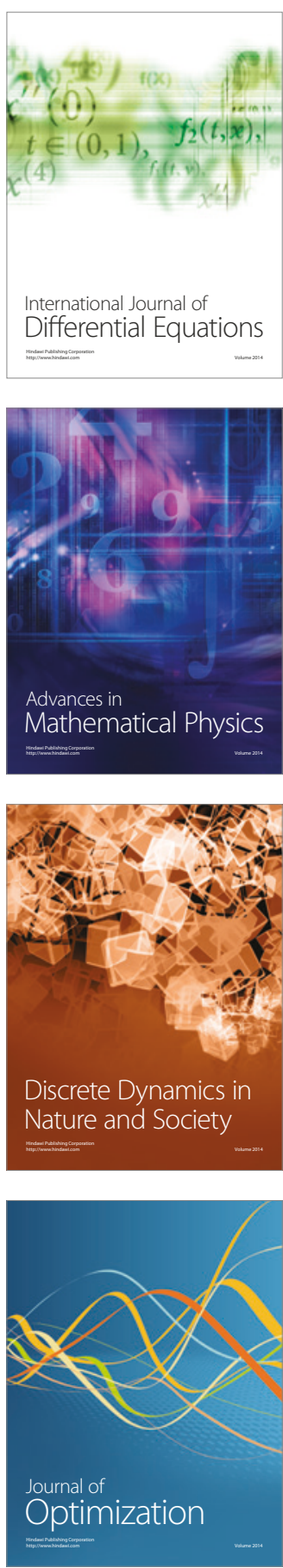